\title{
Oral manifestations in chronic neutropenia in adults: a case report
}

\author{
Gabriela Alacarina Farina ${ }^{1}$, Stefanie Thieme Perotto ${ }^{1}$ and Maria Cristina Munerato ${ }^{2 *}$ \\ ${ }^{1}$ Resident, Dentistry, Stomatology, Dentistry College, Universidade Federal do Rio Grande do Sul (UFRGS), Porto Alegre, Brazil \\ ${ }^{2}$ Stomatology, Associate professor, Department of Conservative Dentistry, Dentistry College, Universidade Federal do Rio Grande do Sul (UFRGS), Porto Alegre, Brazil; \\ Stomatology Unit, Hospital de Clínicas de Porto Alegre (HCPA), Porto Alegre, Brazil
}

\begin{abstract}
Chronic Neutropenia (CNP) is a primary blood disorder characterized by very low neutrophil levels every 3 to 4 weeks. Manifesting as oral ulcers, fever, lymphadenopathy, indisposition, diarrhea, and severe infections, CNP is a systemic condition that may induce the emergence of oral ulcers whose clinical aspects are sometimes mistaken for a series of other diseases. In addition, the oral manifestation of CNP may be the first event to lead a patient to look for professional healthcare. This case report describes CNP in an adult male patient with oral lesions recurring for two years and who was diagnosed by his dentist.
\end{abstract}

\begin{abstract}
Abbreviations: $\mathrm{BD}$ : Behcet's disease, $\mathrm{CBC}$ : complete blood count, CEP-HCPA : Committee for Ethics in Research of HCPA, CNP : chronic neutropenia, HCPA : Hospital de Clínicas de Porto Alegre, HIV : human immunodeficiency virus, IBD : inflammatory bowel disease, NP : neutropenia, RAS : recurrent aphthous stomatitis, ROU : recurrent oral ulceration, SLE : systemic lupus erythematosus.
\end{abstract}

\section{Introduction}

Defined as a persistent decrease in absolute neutrophil counts, NP may be diagnosed on its own or with thrombocytopenia and anemia [14]. Though most NP cases are acquired manifestations of the disease, it may also be congenital. The congenital forms of NP described in adults were categorized as constitutional, ethnic, benign familial, and cyclic. More numerous compared with congenital NP, the acquired forms of the disease may manifest as an autoimmune condition or occur associated with infections, malignant neoplasias, and nutritional deficiencies or even be induced by drugs $[1,4,5]$. In severe cases NP may cause generalized infection and require hospitalization [5].

The accurate diagnosis of CNP in adults is a challenge that calls for exhaustive investigation. The first steps in this inquiry are the analysis of medical records and family history details followed by the evaluation of recurrent infections, high fever, and oral lesions $[1,2,4,5]$. In less urgent cases this assessment is based on neutrophil counts taken three times a week for 6 to 8 weeks. These data provide an insight into the pattern (regular or irregular) and severity (light, mild, or severe) of CNP, helping detect changes in the other figurative elements of blood as well $[1,5]$. Interestingly, it may be useful to keep a detailed written record of signs and symptoms in the effort to find the likely associations between changes in oral health and fever, infections, and the drop in circulating neutrophil counts [1].

In its more severe forms, CNP patients experience neutrophil counts below 500 accompanied by crater-like, hemorrhagic, painful lesions both on the oral mucosa and the tongue. Gingivitis manifesting as erosive lesions with pain and bleeding is also observed. Importantly, CNP may evolve to diffuse lesions along the whole intestinal tract that in turn may be come along with diarrhea and abdominal pain. It has been shown that the significant decrease in circulating neutrophils means that the symptoms of these infections in CNP patients are less evident, with no suppuration or fluctuation [1,2]. This immunosuppression may also lead to secondary infection of these lesions, promoting the hematogenous spread of microorganisms and expanding treatment times.

As a rule CNP is diagnosed by medical doctors. But since the presence of oral ulcers may be the earliest manifestation of the disease, in many cases the dentist will actually be the first health professional to detect the disorder. For this reason, the accurate differential diagnosis of ROU is an important factor in the definition of the best treatment method prescribed by a dentist.

The present study describes the diagnosis of CNP by a dentist in an adult male patient with ROU. A literature review about ROU was carried out in the CAPES, Scielo, Lilacs, and Pubmed databases using the keywords oral manifestations, chronic neutropenia, adults, recurrent oral ulcerations, and differential diagnosis. To improve quality of research, only papers published in 2010 and after were included.

Data were collected using a form previously analyzed and approved by the CEP-HPCA according to official regulations issued by the Ministry of Health of Brazil. The form was documented by CEP-HPCA

${ }^{\star}$ Correspondence to: Maria Cristina Munerato, Departamento de Odontologia Conservadora - Faculdade de Odontologia - UFRGS, Rua Ramiro Barcelos, 2492 - Porto Alegre - Rio Grande do Sul - Brazil, CEP 90035-000, E-mail: mcmunerato@gmail.com

Key words: neutropenia; oral lesions; adults

Received: March 08, 2018; Accepted: March 19, 2018; Published: March 21, 2018 
under number 160128. The patient signed an informed consent form authorizing the use of his medical record and other documents.

\section{Case report}

A 59-year-old African-American male visited the Stomatology Unit of HCPA complaining of oral ulcers that had been recurring for two years. In addition, the patient reported fever and diarrhea concomitant with the lesions. Medical record included current treatment of an anal fistula, though the patient was not taking any drug continuously. Smoker for 40 years, he also consumes alcoholic beverages sporadically and had been referred to a gastroenterologist with suspected celiac disease, which was not confirmed.

Examination of the oral cavity revealed a $25-\mathrm{mm}$ painful, hemorrhagic, crater-like ulcer with necrosed center on the left side of the dorsum of tongue (Figure 1a) that posed feeding and speaking difficulties. Ulcers were also present on lips and on the right jugal mucosa (Figure 1d). These ulcers were recurring every 3 to 4 weeks and healed slowly (Figures $1 \mathrm{~b}$ and $1 \mathrm{c}$ ). The patient had been treated for ROU with dexamethasone $4 \mathrm{mg}$ weekly for six months, to no effect. Due to the large size of oral ulcers no topical medication was prescribed. The patient was given systemic corticosteroid only.

During the consultation the patient produced CBCs taken in the previous 12 months. The results indicated a decrease in circulating neutrophil counts and in total leukocytes in the period. Also, platelet counts were between 100,000 and 140,000 platelets per microliter of blood in all tests. Together, these findings indicated an association between $\mathrm{CNP}$ and mild thrombocytopenia. Oral lesions were considered the outcome of severe NP and the patient was referred to a hematologist for an investigation of the origin of the disease. The patient was also followed-up by the stomatologist.

In the six months between referral and the first appointment with the hematologist, oral ulcers manifested recurrently, accompanied by fever and diarrhea. The stomatological approach was based on chlorhexidine gluconate $0.12 \%$ used as mouthwash to prevent the secondary contamination of ulcers, providing chemical control of bacterial biofilm in the oral environment. Temporary bacteremia is common in the oral cavity and may increase the risk of generalized infection in patients with severe NP. In the time period between referral and first appointment with the hematologist, we requested a weekly $\mathrm{CBC}$ as a tool to identify the periods of NP and thus manage oral manifestations and prevent opportunistic infections in the oral cavity. Although the literature suggests $\mathrm{CBCs}$ three times a week, the patient only had one a week due to commuting difficulties.

After the first CBC a biopsy of bone marrow was conducted (Figures 2 and 3 ) and exams were carried out to investigate autoimmune and infectious diseases that may be involved in CNP (Figure 4). Subsequently to the first appointment with the hematologist, a bone marrow biopsy was conducted followed by exams needed for differential diagnosis.

The $\mathrm{CBC}$ results indicated agranulocytosis, and the patient was given a prescription of filgrastim $300 \mathrm{mcg}$ three times a week for two months. At the follow-up appointment he reported that the oral ulcers had disappeared completely, and the prescription was changed to filgrastim $300 \mathrm{mcg}$ once a week. CBC results improved after treatment, with increased neutrophil counts to levels within the normal immunocompetence range. However, platelet counts remain at between 100,000 and 140,000 platelets per microliter of blood, indicating mild thrombocytopenia.

\section{Discussion}

ROU manifests concomitantly with several diseases, like IBD, $\mathrm{BD}$, nutritional and vitamin deficiencies, infections, autoimmune conditions, and blood disorders. Although it is the most common kind of oral ulcer, RAS is nonspecific, which requires stomatological evaluation in the differential diagnosis of oral diseases and implementation of appropriate treatment [6-8].

In the present case report the patient had multiple lesions that emerged every 3 to 4 weeks. These lesions were mainly painful, craterlike, hemorrhagic ulcers with necrosed center associated with fever and diarrhea. No other kind of oral lesion was observed and, due to diarrhea, the differential diagnosis included IBD, which was ruled out after examination by a gastroenterologist based on endoscopy

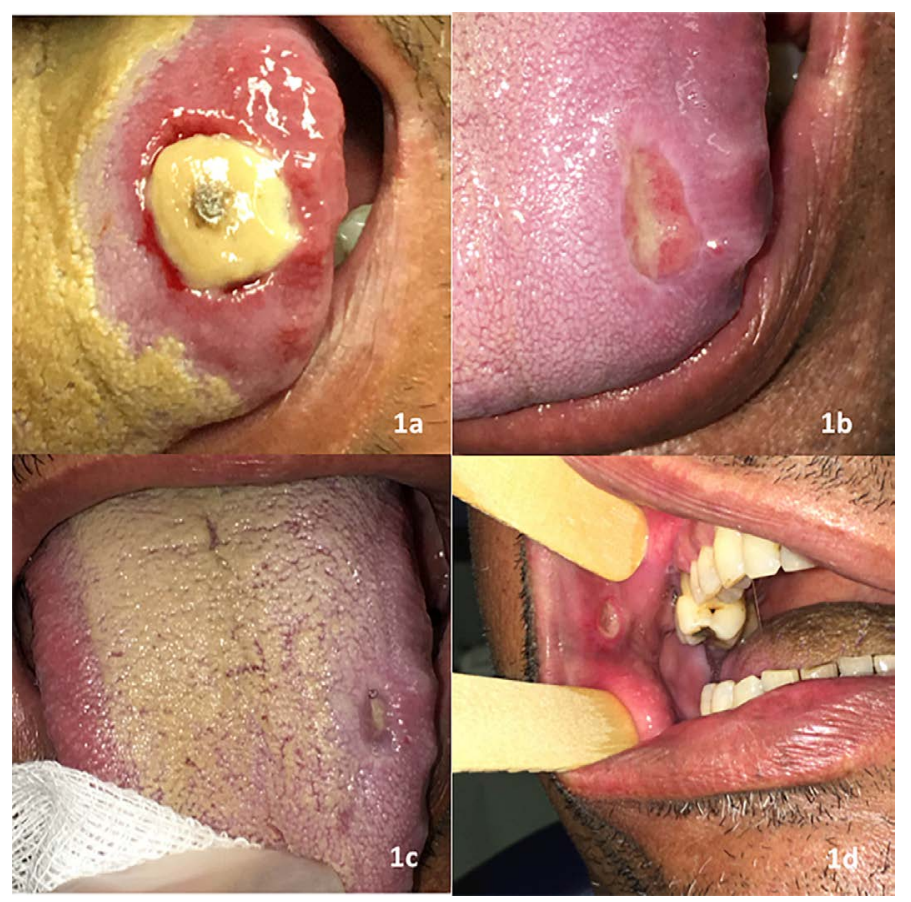

Figure 1. (a)Extensive crater-like lesion on the left side of the dorsum of tongue, necrosed center and hemorrhage; (b) and (c) the same legions after 15 and 30 days, respectively; (d) crater-like lesion on the right jugal mucosa with no inflammatory response

Bone marrow biopsy:

Hypercellular bone marrow with elements of the three series and lymphocytosis.

No mature neutrophils were identified.

Bone marrow exam:

Cellularity: reduced

Neutrophilic granulocytes: rare

Note: rare promyelocytes (5\%) and rare myelocytes $(6 \%)$

Erythroid cells: well represented

Megakaryocytes: well represented

Myeloid/erythroid ratio: $0.2 / 1$

Lymphocytes: $39.0 \%$

Plasmocytes: $5.0 \%$

Monocytes: $1,0 \%$

Basophils: $0.0 \%$

Blastocytes: $3.0 \%$

Foreign cells in bone marrow: negative

Note: mildly hypocellular bone marrow, markedly hypoplastic neutropoiesis, poorly differentiated precursors only, megakaryopoiesis and erythropoiesis with numerous precursors.

Compatible with the informed clinical diagnosis (agranulocytosis).

Figure 2. Results of the bone marrow biopsy 


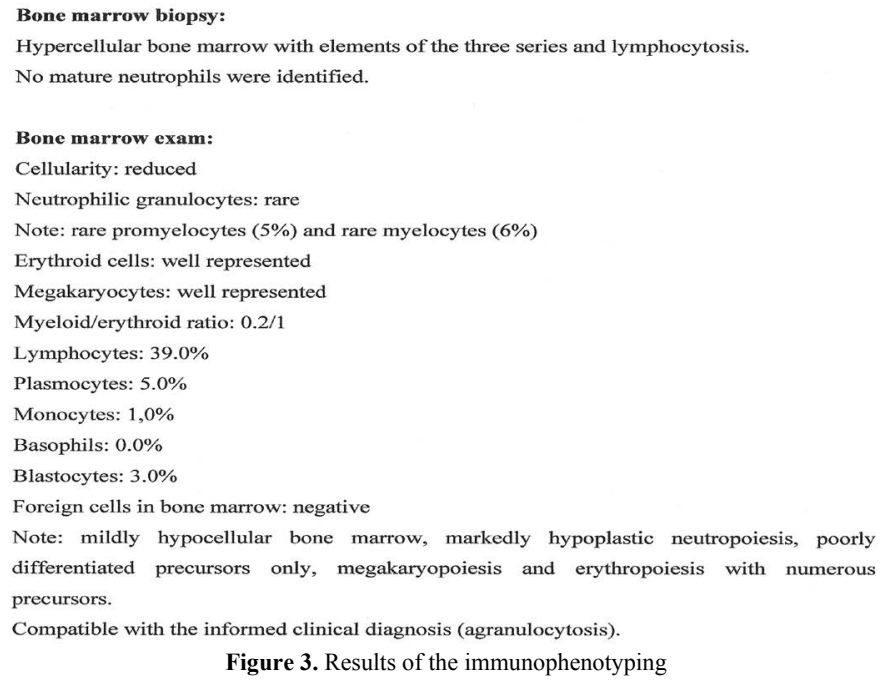

Compatible with the informed clinical diagnosis (agranulocytosis).

Figure 4. Results of the exams carried out during the diagnosis of the case studied

and colonoscopy findings. Patients with Chron's disease, ulcerative rectocolitis, and celiac disease may also present similar ulcers as observed in RAS in addition to other changes in the oral mucosa, like hyperplastic cobblestone lesions and angular cheilitis [6,8-12].

BD may also present oral ulcers similar to ROU before other manifestations, which include recurrent genital lesions, eye lesions, and skin ulcers $[6-9,11-13]$. In the present case report, the patient had no ophthalmological conditions but presented one single genital ulcer episode. Despite that, a pathergy test and ophthalmological exams were requested to rule out $\mathrm{BD}$ as a cause of oral ulcers. The findings afforded to conclude that the patient did not meet BD criteria.

Nutritional and vitamin deficiencies (iron, vitamin B complex, and zinc) may induce the emergence of ulcers on the mucosa, which would respond favorably to treatment with supplementation [6-9]. In the present case report the levels of ferritin and vitamin B12 were normal, ruling out this hypothesis.

Both autoimmune and infectious diseases may manifest as multiple lesions on the oral mucosa. Similar lesions to those observed in RAS are diagnosed in HIV-positive patients, though with no recurrence. HIV may also induce the manifestation of NP, when oral lesions may be mistaken for nonspecific ulcers in the oral mucosa. The CBC carried out as of the emergence of oral lesions is a useful tool in the differential diagnosis of diseases in these HIV-positive individuals. Since the patient in the present study is HIV-negative, this etiology of CNP was ruled out.

Secondary syphilis may also manifest as multiple oral lesions [8,11,13-18]. Oral tuberculosis sometimes occurs with single or multiple ulcers $[19,20]$. In the present case report, the results of tests to exclude infectious diseases were negative. The patient did not undergo the Mantoux test because it was not available in the country at that time. Similarly, he could not produce sputum for analysis, and X-rays of lungs showed the presence of a calcified $0.8-\mathrm{cm}$ nodule on the middle third of the left lung. Since the patient has been a smoker for 40 years, this radiographic finding may be an outcome of previous lung disease and was not considered relevant.

The viral etiology of oral lesions also have to be considered in the differential diagnosis of CNP, such as those observed in infections with cytomegalovirus, herpes simplex virus, varicella zoster virus, Epstein-Barr virus, and Coksackies virus [1,6,8,12,13,18,21]. Deep mycoses may also manifest as chronic as well as non-ROU [22]. Due to this characteristic these mycoses were not included in the differential diagnosis in this case report.

The main autoimmune diseases that may induce NP with oral lesions are rheumatoid arthritis, SLE, Sjogren's syndrome, and multiple sclerosis $[8,17]$. More specifically, SLE may manifest first as oral mucosa lesions, and should be included in the differential diagnosis of oral ulcers $[6,9]$.

In the present case report, the patient produced the CBCs carried out in the months preceding his first appointment with the Stomatology Service of HPCA. Considered in view of the complaints made by him, these findings supported CNP as the main cause of ROU. Fever and diarrhea associated with these lesions also indicated that ROU could not be the definitive diagnosis, since the condition is not accompanied by signs in systems or organs other than the oral cavity. Moreover, the patient had been unsuccessfully treated for RAS with dexamethasone $4 \mathrm{mg}$ for six months.

In a case series that included 108 cases of severe primary CNP in adults, $45.5 \%$ of the patients had similar lesions to RAS, while $60.2 \%$ had bacterial infections [23]. Oral ulcerative lesions are more frequently detected in individuals with neutrophil counts below 500 cells per microliter, that is, in severe PN. These patients may also present diffuse lesions on the gastrointestinal tract $[1,2]$. which could explain the diarrhea and abdominal pain described by the patient in the present case report.

Fever, diarrhea, anal fistula, and one single lesion on the lingual mucosa have been reported in a patient with NP. ${ }^{17}$ These were also diagnosed in the patient in the present case report, except for the fact that he had multiple oral mucosa lesions. Because of immunosuppression due to significantly low neutrophil counts, the symptoms of infections in these patients less evident, with no suppuration and fluctuation $[1,2]$.

Another hypothesis that should be considered is the occurrence of lesions in neutropenic patients with lymphoproliferative diseases [24]. In the present case report, bone marrow was biopsied, but the exams carried out did not reveal this kind of disease.

NP induced by pharmaceuticals, especially in patients undergoing anticancer chemotherapy, is accompanied by fever and oral mucosa 
lesions [25]. In the present case report the patient had no diagnosed malignant disease, was not undergoing chemotherapy, and did not use any medical drug continuously.

The use of mouthwash with chlorhexidine is indicated to control the bacterial flora in the oral cavity and therefore prevent secondary infection of crater-like lesions that are typically observed in NP and could lead to sepsis $[24,26]$.

\section{Conclusion}

$\mathrm{CNP}$ is one of the many systemic conditions that may induce the emergence of oral lesions whose clinical aspects can be mistaken for a series of other diseases. Also, the oral manifestation of NP sometimes is the first sign that a patient should look for medical assistance. In such scenario, dentists have to be familiar with the interpretation of $\mathrm{CBC}$ findings, which would be the first exam to be requested to a patient with oral lesions accompanied by fever and gastrointestinal symptoms.

\section{Acknowledgements}

The authors are grateful to the patient, who gave consent for this study to be carried out.

\section{Conflict of interests}

None.

\section{References}

1. Boxer LA (2012) How to approach neutropenia. Hematology Am Soc Hematol Educ Program 2012: 174-182. [Crossref]

2. Dale DC (2016) How I diagnose and treat neutropenia. Curr Opin Hematol 23: 1-4. [Crossref]

3. Estcourt LJ, Stanworth S, Doree C, Blanco P, Hopewell S, et al. (2015) Granulocyte transfusions for preventing infections in people with neutropenia or neutrophil dysfunction Cochrane Database Syst Rev 6: CD005341. [Crossref]

4. Newburger PE, Dale DC (2013) Evaluation and management of patients with isolated neutropenia. Semin Hematol 50: 198-206. [Crossref]

5. Gibson C, Berliner N (2014) How we evaluate and treat neutropenia in adults. Blood 124: 1251-1258. [Crossref]

6. Talacko AA, Gordon AK, Aldred MJ (2010) The patient with recurrent oral ulceration. Aust Dent J 55 Suppl 1: 14-22. [Crossref]

7. Vaillant L, Samimi M (2016) Aphthous ulcers and oral ulcerations. Presse Med 45: 215-226. [Crossref]

8. Lehman JS, Rogers RS (2016) Acute oral ulcers. Clin Dermatol 34: 470-474. [Crossref]
9. Mays JW, Sarmadi M, Moutsopoulos NM (2012) Oral manifestations of systemic autoimmune and inflammatory diseases: diagnosis and clinical management. $J$ Evid Based Dent Pract 12: 265-282. [Crossref]

10. Pereira MS, Munerato MC (2016) Oral Manifestations of Inflammatory Bowel Diseases: Two Case Reports. Clin Med Res 14: 46-52. [Crossref]

11. Siu A, Landon K, Ramos DM (2015) Differential diagnosis and management of oral ulcers. Semin Cutan Med Surg 34: 171-177. [Crossref]

12. Akintoye SO, Greenberg MS (2014) Recurrent aphthous stomatitis. Dent Clin North Am 58: 281-297. [Crossref]

13. CEDEF (2012) Item 343--Ulceration or erosion of the oral and/or genital mucosa. Ann Dermatol Venereol 139: A239-243. [Crossref]

14. Feller L, Khammissa RA, Wood NH, Meyerov R, Pantanowitz L, et al. (2012) Ora ulcers and necrotizing gingivitis in relation to HIV-associated neutropenia: a review and an illustrative case. AIDS Res Hum Retroviruses 28: 346-351. [Crossref]

15. Watts PJ, Greenberg HL, Khachemoune A (2016) Unusual primary syphilis Presentation of a likely case with a review of the stages of acquired syphilis, its differential diagnoses, management, and current recommendations. Int J Dermatol 55: 714-728. [Crossref]

16. Seibt CE, Munerato MC (2016) Secondary syphilis in the oral cavity and the role of the dental surgeon in STD prevention, diagnosis and treatment: a case series study. Braz J Infect Dis 20: 393-398. [Crossref]

17. Kurago ZB, Kerr AR, Narayana N (2011) Clinical pathologic conference case 5 agranulocytosis. Head Neck Pathol 5: 286-291. [Crossref]

18. Schemel-Suárez M, López-López J, Chimenos-Kústner E (2015) Úlceras orales: diagnóstico diferencial y tratamiento. Med Clin (Barc) 145: 499-503.

19. Krawiecka E, Szponar E (2015) Tuberculosis of the oral cavity: an uncommon but still a live issue. Postepy Dermatol Alergol 32: 302-306. [Crossref]

20. de Souza BC, de Lemos VM, Munerato MC (2016) Oral manifestation of tuberculosis: a case-report. Braz J Infect Dis 20: 210-213. [Crossref]

21. Parent D (2016) Oral viral infections. Presse Med 45: 196-214. [Crossref]

22. Krishnan PA (2012) Fungal infections of the oral mucosa. Indian J Dent Res 23: 650659. [Crossref]

23. Fontbrune S, Moignet A, Beaupain V, Suarez F, Galicier L, et al. (2015) Severe chronic primary neutropenia in adults: report on a series of 108 patients. Blood 126: 1643-50. [Crossref]

24. Arvanitidou IE, Nikitakis NG, Sklavounou A (2011) Oral manifestations of T-cell large granular lymphocytic leukemia: a case report. J Oral Maxillofac Res 2: e4. [Crossref]

25. Ataseven E, Bengoa SY, Ören H (2016) Stevens-Johnson Syndrome/Toxic Epidermal Necrolysis Should Be Kept in Mind in Children with Febrile Neutropenia, Oral Cavity Lesions, and Skin Rash. Turk J Hematol 33: 170-171. [Crossref]

26. Gomes RF, Munerato MC (2016) The Stomatological Complications of DiamondBlackfan Anemia: A Case Report. Clin Med Res 14: 97-102. [Crossref]

Copyright: (C2018 Farina GA. This is an open-access article distributed under the terms of the Creative Commons Attribution License, which permits unrestricted use, distribution, and reproduction in any medium, provided the original author and source are credited. 